Communication, technologies et développement

5 | 2018

Le numérique et le développement des Suds

\title{
Langues et médias en Afrique noire francophone
}

\section{Venant Eloundou Eloundou}

\section{OpenEdition}

\section{Journals}

Édition électronique

URL : http://journals.openedition.org/ctd/423

DOI : $10.4000 /$ ctd. 423

ISSN : 2491-1437

\section{Éditeur}

Chaire Unesco Pratiques émergentes en technologies et communication pour le développement

\section{Référence électronique}

Venant Eloundou Eloundou, "Langues et médias en Afrique noire francophone », Communication, technologies et développement [En ligne], 5 | 2018, mis en ligne le 02 janvier 2018, consulté le 24 septembre 2020. URL : http://journals.openedition.org/ctd/423 ; DOI : https://doi.org/10.4000/ctd.423

Ce document a été généré automatiquement le 24 septembre 2020

Communication, technologies et développement 


\title{
Langues et médias en Afrique noire francophone
}

\author{
Venant Eloundou Eloundou
}

\section{RÉFÉRENCE}

Louis Martin Onguéné Essono, Analyse (socio) linguistique et didactique, Paris,

Connaissances et Savoirs, col. Lettres et Langues, 2017, 347 p.

1 Les quinze articles qui forment l'ouvrage sont le fruit d'une longue activité de recherches menées pendant près de 25 ans par Louis Martin Onguéné Essono. Elles ont pour centre de gravitation les médias et les langues en Afrique noire francophone. Le contenu des articles, focalisés dans ce volume sur les sciences de l'information et de la communication, permet de ressortir trois principaux intérêts heuristiques.

2 Le premier retrace le parcours de la naissance de la presse écrite au Cameroun. L'auteur expose son évolution avant et après l'indépendance. Il scrute les différentes mutations qu'elle a subies. De 1903 à 1971, l'on a connu la floraison de la presse écrite en langue nationale. Qui aura fortement contribué à « la formation et l'unification de clans politiques, pour communiquer les informations, excluant leur décodage par les non locuteurs, [et] rassembler plus facilement les ressortissants d'une même tribu " (p. 88). Chaque tribulangue voulait ainsi gérer l'information sociopolitique, chrétienne ou culturelle au niveau endogène ou intracommunautaire. C'est le cas de Mulée Nguea (Le guide, en duala), Nleb bekristen (Le conseiller des chrétiens en ewondo), etc.

Louis Martin Onguéné Essono analyse ensuite les causes de la déchéance de cette presse africaine en langues nationales, notamment le manque de politique linguistique éducative efficiente pour l'enseignement de ces langues à l'école, la volonté des acteurs politiques des pays nouvellement indépendants à œuvrer pour l'unité linguistique des populations hétérogènes, les politiques d'imposition des langues exogènes initiées par le colonisateur, au détriment des langues nationales. 
4 Le deuxième intérêt du livre porte sur la configuration du français utilisé dans la presse en Afrique noire francophone. Partant du constat selon lequel le français pratiqué en Afrique par les Africains comporte de nombreux traits de démarcation par rapport au français de France, l'auteur pose que la presse écrite constitue un lieu d'actualisation de la variation de cette langue. Son objectif primordial est d'analyser les mutations médiatiques africaines contemporaines impliquant les transformations de la langue. Les terrains de cette observation sont la presse publique, la presse privée et les TIC. Il met aussi en lumière les différents écarts linguistiques de divers ordres : lexical (avec le phénomène de l'emprunt aux langues nationales), la créativité lexicale (c'est le cas de médiocritisation, p. 125), des écarts morphosyntaxiques, et prioritairement l'usage des prépositions (ex. Moukoko $\mathrm{M}$ insiste à ce que l'information soit annoncée, p. 106) et des altérations lexico-sémantiques, marquées par les procédés de désémantisation et de resémantisation (ex. le plat de poisson coûte celui du gibier dans les circuits d'Hevecam, p. 268). La conséquence de l'usage d'une norme du français endogène est que l'éventail de la réception se retrouve réduit. Seule la communauté francophone du Cameroun peut comprendre le message véhiculé par cette presse. Le lexique étranger au français standard "déclenche un circuit lexical nouveau, qui reformule une lexigénie fondée sur la société culturelle, utilisatrice d'un dialecte donné, et qui l'impose et la superpose à l'ordre linguistique du français imposé et subi» (p. 135). Pour lui, les écrivains-journalistes sont dotés d'une L1 qui se répercute sur leur français » (p.21). Ce qui est à l'origine des subgrammaires.

5 L'auteur ne se contente pas d'analyser ces écarts linguistiques. Il sonde aussi les causes qui favorisent leur actualisation dans les discours des journalistes de la presse écrite. Parmi ces causes, il identifie : le contexte sociolinguistique des pays de l'Afrique noire francophone, marqués par le multilinguisme ; l'influence des L1 sur la L2 (le français) dont la conséquence est qu'il devient « difficile de parvenir à un bilinguisme réel qui permet au rédacteur moyen de la presse écrite camerounaise de s'élever au-dessus de la culture et de sa langue pour s'exprimer comme un locuteur natif du français» (p. 116). Outre ces deux causes, Louis Martin Onguéné Essono mentionne la scolarisation et estime qu'elle est bricolée; ce qui ne permet pas aux journalistes d'avoir une compétence linguistique avérée pour s'exprimer convenablement en français standard. La scolarisation de ces derniers étant bricolée, la norme est bafouée par méconnaissance ou par ignorance. Face à cette situation où la langue française est déformée, l'auteur pense que les journalistes devraient régulièrement utiliser le dictionnaire pour éviter «la dialectisation du français, source de tous les dangers » (p. 135).

6 L'ultime portée majeure de l'ouvrage est l'exploitabilité des TIC dans le processus des TICE. Dans ce sillage, l'auteur présente les résultats des expériences de l'application des TIC à l'enseignement/ apprentissage du français à l'école (cf. pp. 183 et 201). Il démontre que les TIC constituent un outil efficace pour améliorer considérablement le processus didactique du français au Cameroun. Il analyse une expérience de l'autoapprentissage du vocabulaire par des élèves grâce à un ordinateur doté d'un dictionnaire électronique. La saisie d'une production d'écrit permet à l'élève de détecter des écarts lexicaux, puisqu'un ordinateur ayant des dictionnaires « codifiés peut signaler la transgression de la syntaxe orthographique ou morphologique» (p. 198). "Le problème inhérent à cet outil concerne la dérivation et la néologie » (p. 198). Un apprenant qui utilise un mot non attesté dans le lexique du français le sait grâce au signalement de 
l'ordinateur. De même, un mot mal saisi peut être corrigé de manière automatique. Par cet outil, l'élève parvient à mesurer ses compétences lexicales.

7 Sous un autre angle, les productions de la presse écrite peuvent constituer un corpus efficace pour l'observation du fonctionnement de la langue française. Son exploitation dans une classe de français est tout à fait possible. En effet, dans l'optique d'utiliser des documents authentiques en lieu et place des morceaux choisis, l'auteur soutient que les enseignants gagneraient à manipuler avec leurs élèves les énoncés de la presse écrite pour étudier certains items de la langue française, tels que la grammaire des textes qui implique les problèmes liés à la cohérence et la cohésion textuelle, la morphologique lexicale et la sémantique.

Par ailleurs, il insiste sur l'intervention de l'Internet dans le processus d'enseignement/ apprentissage des disciplines, et notamment la langue française. Internet constitue ainsi un canal de vulgarisation des savoirs, un puits où devraient s'abreuver non seulement les enseignants, mais également les apprenants orientés. Dès lors, la didactique change avec l'apport du multimédia. L'enseignant devient un guide, un facilitateur et l'apprenant, un acteur qui ne subit plus les savoirs. Il n'est plus un réceptacle de savoirs. Il participe à sa formation. Compte tenu de ces apports des TIC (p. 201), Louis Martin Onguéné Essono propose des solutions en vue de son implémentation dans le secteur éducatif de l'Afrique noire francophone, non sans rappeler les acquis. Parmi ces propositions, il mentionne : la poursuite de la création des centres de ressources multimédia dans des établissements scolaires et universitaires, la facilitation de l'acquisition des appareils multimédias, l'accès à l'Internet, la mise en ligne des cours et l'application des TICE à tous les niveaux de formation.

9 Malgré la pertinence de cet ouvrage, l'on regrettera deux insuffisances. Les analyses réalisées ne prennent pas véritablement leur source sur la presse écrite de toute l'Afrique noire francophone, comme l'annonce le titre principal. La plupart des exemples sont tirés de la presse écrite du Cameroun. On le remarquera d'ailleurs lorsque l'auteur dresse la genèse de la presse écrite (p. 88-89), il ne fait pas allusion à la presse sénégalaise, gabonaise, etc. On peut donc supposer que sa démarche a été hypothético-déductive.

10 Le second regret est la complexité de l'orientation heuristique des analyses. D'une part, on est tenté de dire que l'auteur a mené des réflexions grammaticales sur le français de la presse écrite au Cameroun, puisqu'il privilégie la norme du français et non des normes. Les écarts constatés constituent des transgressions à cette norme. Par ailleurs, il oppose le français de la presse écrite camerounaise à celui des journalistes français ayant pour langue première le français. Dès lors, un journaliste français devient un locuteur idéal ; pourtant, même en France métropolitaine, les mutations du français sont perceptibles. Le français des natifs constitue pour l'auteur une référence, un modèle vers lequel devrait tendre tout locuteur scolarisé. On retrouve à ce niveau le locuteur idéal postulé par Chomsky. Il aurait pu faire des incursions dans la presse française pour mesurer et même comparer les formes d'altération du français.

11 D'autre part, les réflexions de ce volume ont une allure sociolinguistique. A côté de la posture puriste de Louis Martin Onguéné Essono, la thèse de l'enrichissement ou même de la dynamique du français n'est pas exclue. Tantôt ces écarts décrits trouvent leur fondement dans l'apparition de nouvelles réalités que la langue française doit prendre en charge, tantôt le français de la presse écrite au Cameroun est saisi comme un 
résultat de l'échec de l'enseignement du français et des lacunes curriculaires de formation de journalistes, tantôt les écarts linguistiques participent de l'enrichissement du français en Afrique noire. Cette complexité semble avoir deux sources. L'auteur a sans doute été influencé par sa profession universitaire: grammairien et défenseur de la norme et non des normes, il a eu une saisie agrammaticale des productions de la presse camerounaise et a fait des propositions en vue d'améliorer considérablement sa qualité. En même temps, il n'a pas été insensible aux travaux de sociolinguistique francophone engagés depuis les années 1970, travaux qui démontrent que la langue française ne saurait être appréhendée en Afrique sous le seul prisme structuraliste ou normatif, puisque cette langue s'est fortement acclimatée dans des nouvelles écologies, donnant lieu aux endonormes qu'il faudrait dorénavant promouvoir dans le cadre d'une francophonie plurielle. Voilà sans doute pourquoi l'ouvrage porte comme sous-titre, Analyse (socio) linguistique et didactique. Dès lors, la démarche heuristique de l'auteur montre que la grammaire en tant qu'outil œuvrant pour l'harmonisation des usages, en en valorisant certains, ne devrait plus ignorer les pratiques langagières dans la société, les valeurs et les représentations qu'elles drainent.

Complété avec beaucoup de bonheur le très bel ouvrage paru en 2013 aux éditions Clé de Yaoundé, au Cameroun, Dynamique du français dans la presse francophone du Cameroun, ce second livre, préfacé par Marie-Soleil Frère, Langues et médias en Afrique noire francophone, Analyse (socio) linguistique et didactique, dresse un état des lieux des usages linguistiques dans la presse écrite et les apports des TIC aux processus des TICE en Afrique. À cet égard, il ouvre des pistes de réflexions qui demeurent peu ou non explorées : d'où sa portée stimulante. 